\title{
Level of 8-OHdG in drained dialysate appears to be a marker of peritoneal damage in peritoneal dialysis
}

This article was published in the following Dove Press journal: International Journal of Nephrology and Renovascular Disease 28 December 201I

Number of times this article has been viewed

\section{Yoshiyuki Morishita Minami Watanabe \\ Ichiro Hirahara \\ Tetsu Akimoto \\ Shigeaki Muto \\ Eiji Kusano}

Division of Nephrology, Department of Medicine, Jichi Medical University, Tochigi, Japan
Correspondence: Yoshiyuki Morishita Division of Nephrology, Department of Medicine, Jichi Medical University, 33 I I-I Yakushiji, Shimotsuke City, Tochigi 329-0498, Japan

Tel +8I 285587346

Fax +8I 285444869

Email ymori@jichi.ac.jp
Purpose: Peritoneal dialysis (PD) is a successful renal replacement therapy; however, long-term PD leads to structural and functional peritoneal damage. Therefore, the monitoring and estimation of peritoneal function are important in PD patients. Oxidative stress has been implicated as one possible mechanism of peritoneal membrane damage. The aim of this study was to evaluate the association between an oxidative stress marker, 8-hydroxydeoxyguanosine (8-OHdG), and peritoneal damage in PD patients.

Methods: The authors evaluated $8-\mathrm{OHdG}$ in drained dialysate by enzyme immunoassay to investigate the association between $8-\mathrm{OHdG}$ and solute transport rate estimated by peritoneal equilibration test and matrix metalloproteinase-2 (MMP-2) level in 45 samples from 28 PD patients.

Results: The 8-OHdG level was significantly correlated with dialysate:plasma creatine ratio $(r=0.463, P<0.05)$ and significantly inversely correlated with $\mathrm{D} / \mathrm{D} 0$ glucose (where $\mathrm{D}$ is the glucose level of peritoneal effluents obtained 4 hours after the injection and D0 is the glucose level obtained immediately after the injection) $(r=-0.474, P<0.05)$. The 8-OHdG level was also significantly correlated with MMP-2 level $(r=0.551, P<0.05)$, but it was not correlated with the age of subjects, the duration of $\mathrm{PD}$, or blood pressure.

Conclusion: The level of 8-OHdG in drained dialysate may be a useful novel marker of peritoneal damage in PD.

Keywords: oxidative stress, solute transport rate, MMP-2, peritoneal equilibration test

\section{Introduction}

Peritoneal dialysis (PD) is a successful renal replacement therapy for end-stage renal disease patients. ${ }^{1,2}$ It is suitable as the first method of renal replacement therapy; however, long-term PD leads to peritoneal damage. ${ }^{3,4}$ This peritoneal damage has been found to be characterized by progressive increase in the thickness of peritoneal membrane, predominantly in the submesothelial collagenous area, declining ultrafiltration loss, and an increased solute transport rate. ${ }^{4-6}$ This damage makes it difficult to continue PD therapy and may occasionally cause encapsulating peritoneal sclerosis (EPS), which is a serious, life-threatening complication in PD patients. ${ }^{7,8}$ Therefore, both monitoring and estimation of peritoneal function are important in PD patients. As an estimator of peritoneal damage, the solute transport rate is usually measured by the peritoneal equilibration test (PET) $;^{9,10}$ however, PET is an invasive and timeconsuming method because it requires blood sampling and it takes half a day for patients. Therefore, it is important to estimate peritoneal damage using a conventional and noninvasive method. 
The mechanisms involved in structural and functional peritoneal changes remain unclear. Oxidative stress has been implicated as one possible mechanism of peritoneal membrane damage. ${ }^{11}$ PD fluid containing high concentrations of glucose has been reported to increase cellular reactive oxygen species (ROS). ${ }^{12}$ In addition, recent studies have reported the beneficial effects of antioxidants on preservation of the structural and functional integrity of the peritoneal membrane. ${ }^{13,14}$ From these lines of evidence, the authors hypothesized that an oxidative stress marker may be a useful marker of peritoneal damage in PD patients. To test this hypothesis, the authors measured an oxidative stress marker, 8-hydroxydeoxyguanosine (8-OHdG), in drained dialysate and investigated the association between the 8-OHdG level and the solute transport rate estimated by PET and matrix metalloproteinase-2 (MMP-2) level, which was reported to be a marker of peritoneal damage in PD patients..$^{15,16}$

\section{Material and methods}

This study was performed in accordance with the Declaration of Helsinki and was approved by the ethics committee of Jichi Medical University. Written informed consent was obtained from all patients.

\section{Subjects}

A total of 45 drained dialysate samples from 28 PD patients (18 males and 10 females, mean age $56.3 \pm 13.1$ years, ranging from 25 to 78 years) were investigated. The patients' initial nephropathies were chronic glomerulonephritis (eleven patients), diabetic nephropathy (eight patients), lupus nephritis (one patient), drug nephropathy (one patient), chronic renal sclerosis (two patients), gout kidney (one patient), and unknown etiology (four patients). Nine patients had a history of peritonitis due to infection with a pathogenic organism and were treated with antibiotics.

\section{PET}

Peritoneal solute transport was assessed with the PET. ${ }^{10}$ Drainage of intra-abdominal fluid was followed by an intraperitoneal injection of $2 \mathrm{~L}$ of $\mathrm{PD}$ fluid containing $2.27 \%-2.5 \%$ glucose. The creatinine $(\mathrm{Cr})$ level of peritoneal effluents obtained 4 hours after the injection (D) was divided by that of plasma (P) to obtain the $\mathrm{D} / \mathrm{P}$ Cr ratio. The glucose level of peritoneal effluents obtained 4 hours after the injection (D) was divided by that obtained immediately after the injection (D0) to obtain the D/D0 glucose ratio.

\section{Laboratory methods}

The concentrations of 8-OHdGH and MMP-2 in the drained dialysate obtained from the PET were measured by enzymelinked immunosorbent assay (ELISA).

\section{The 8-OHdG ELISA}

The Highly Sensitive 8-OHdG Check ELISA kit (Japan Institute for the Control of Aging [JaICA], Nikken SEIL Co, Shizuoka, Japan) was used according to the manufacturer's protocol. Briefly, 8-OHdG monoclonal antibody and $50 \mu \mathrm{L}$ of peritoneal effluents were added to the microtiter plate, which had been precoated with 8-OHdG. Following this, $50 \mu \mathrm{L}$ of reconstituted primary antibody to $8-\mathrm{OHdG}$ was added per well and incubated at $4^{\circ} \mathrm{C}$ overnight. After three washes, the reconstituted secondary antibody was added and incubated at room temperature for 1 hour. After three more washes, substrate was added to each well and incubated at room temperature for 15 minutes. The reaction-terminating solution was then added and the absorbance was read at $450 \mathrm{~nm}$.

Table I Patients' baseline characteristics $(\mathrm{N}=28)$

\begin{tabular}{ll}
\hline Parameter & Statistics \\
\hline Age (years) & $56.3 \pm 13.1$ \\
Gender & \\
Male & 18 \\
Female & 10 \\
BMI (kg/m $\left.{ }^{2}\right)$ & $23.4 \pm 4.3$ \\
Duration of PD (years) & $1.9 \pm 2.1$ \\
Peritonitis history (number of times) & \\
0 & 19 \\
I & 5 \\
2 & 2 \\
3 & $\mathrm{I}$ \\
4 & $\mathrm{I}$ \\
Initial nephropathy & \\
Chronic glomerulonephritis & $\mathrm{II}$ \\
Diabetic nephropathy & 8 \\
Lupus nephritis & $\mathrm{I}$ \\
Drug nephropathy & $\mathrm{I}$ \\
Renal sclerosis & 2 \\
Gout kidney & $\mathrm{I}$ \\
Unknown & 4 \\
SBP (mmHg) & $\mathrm{I}$ \\
DBP (mmHg) & $83.3 \pm 12.8 \pm 27.6$ \\
Hemoglobin (g/dL) & $9.8 \pm 1.3$ \\
Albumin (g/dL) & $3.2 \pm 0.3$ \\
\hline No Age BMl dua &
\end{tabular}

Note: Age, BMI, duration of PD, SBP, DBP, hemoglobin, and albumin data are expressed as the mean plus or minus the standard deviation.

Abbreviations: BMI, body mass index; DBP, diastolic blood pressure; $\mathrm{PD}$, peritoneal dialysis; SBP, systolic blood pressure. 


\section{The MMP-2 ELISA}

MMP-2 ELISA was performed using the Biotrak MMP-2 ELISA kit (GE Healthcare, Piscataway, NJ) according to the manufacturer's protocol. Briefly, standards and samples were incubated in microtiter wells precoated with an anti-MMP-2 antibody. Bound MMP-2 was detected using a peroxidaselabeled Fab' antibody to MMP-2 and any excess was removed by washing and aspiration. The amount of peroxidase bound to each well was determined by addition of a 3,3',5,5' -tetramethylbenzidine substrate. The reaction was stopped and the resultant color was read at $450 \mathrm{~nm}$.

\section{Statistical analysis}

All data are expressed as the mean plus or minus the standard deviation. The means of indicated groups were compared using Student's $t$-test. Relationships between continuous variables were analyzed using Pearson correlation tests or linear regression analysis. Differences with a $P$-value less than 0.05 were considered statistically significant.

\section{Results}

Patient characteristics are presented in Table 1. Their duration of PD was $1.9 \pm 2.1$ years. Their systolic blood pressure (SBP) was $146.5 \pm 27.6 \mathrm{mmHg}$ and diastolic blood pressure (DBP) was $83.3 \pm 12.8 \mathrm{mmHg}$. The patients' hemoglobin and albumin levels were low: hemoglobin was $9.8 \pm 1.3 \mathrm{~g} / \mathrm{dL}$ and albumin was $3.2 \pm 0.3 \mathrm{~g} / \mathrm{dL}$. The $8-\mathrm{OHdG}$ levels in drained dialysate were not significantly different by sex, initial nephropathy (diabetic or nondiabetic), or past history of peritonitis (Figure 1).

\section{Correlation between the 8-OHdG concentration and the peritoneal solute transport rate and MMP-2 level}

The 8-OHdG level in the drained dialysate was significantly correlated with D/P Cr $(r=0.463, P<0.05)$ and significantly inversely correlated with D/D0 glucose $(r=-0.474$, $P<0.05$ ) in PD patients (Figure 2A). The 8-OHdG level in the drained dialysate was also significantly correlated with
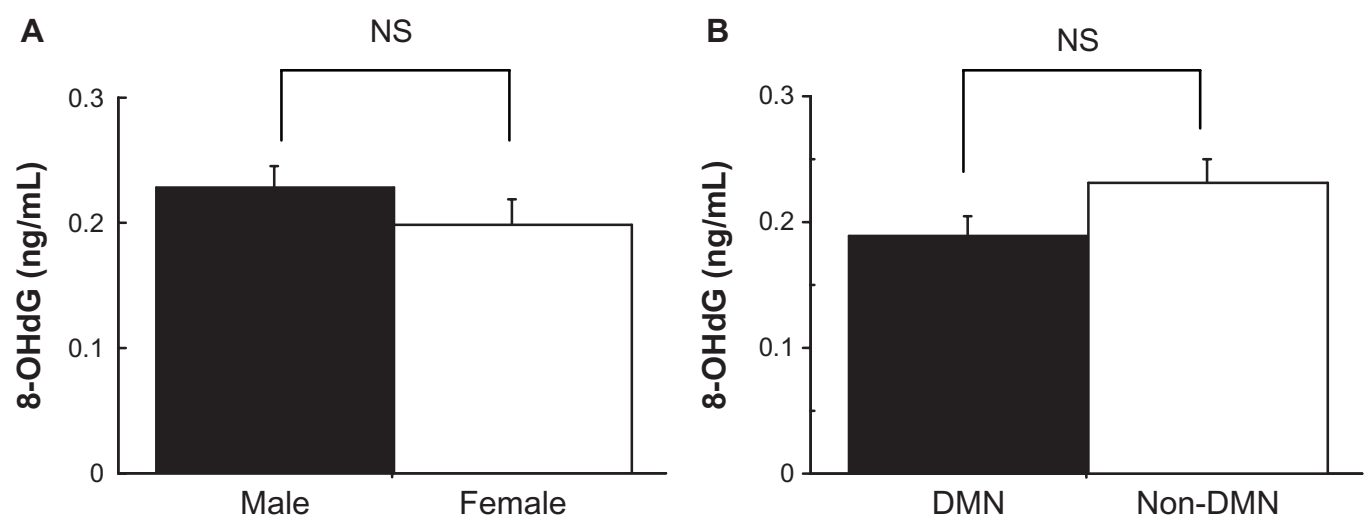

C

NS

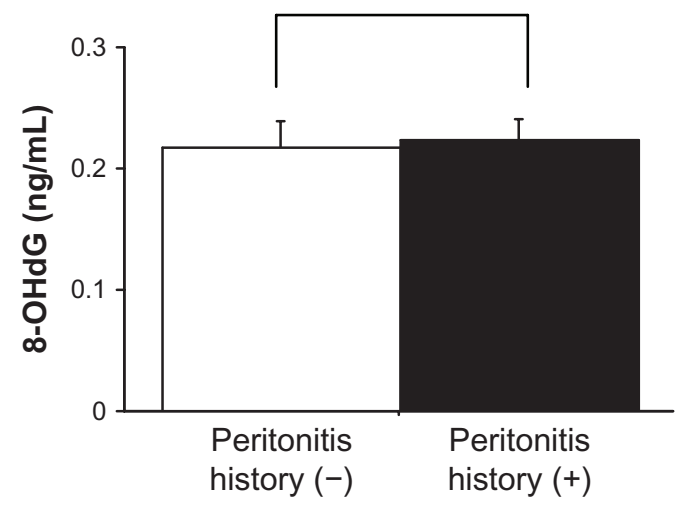

Figure I The level of 8-hydroxydeoxyguanosine (8-OHdG) in drained dialysate in peritoneal dialysis (PD) patients. Comparison of 8-OHdG levels in drained dialysate between $(\mathbf{A})$ males and females, (B) those who had diabetic nephropathy (DMN) and those who did not (non-DMN), and (C) those who had a past history of peritonitis and those who did not.

Note: Columns represent the mean plus or minus standard error.

Abbreviation: NS, no statistical significance. 
A

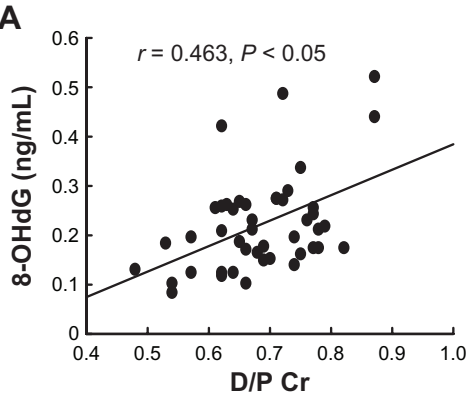

B
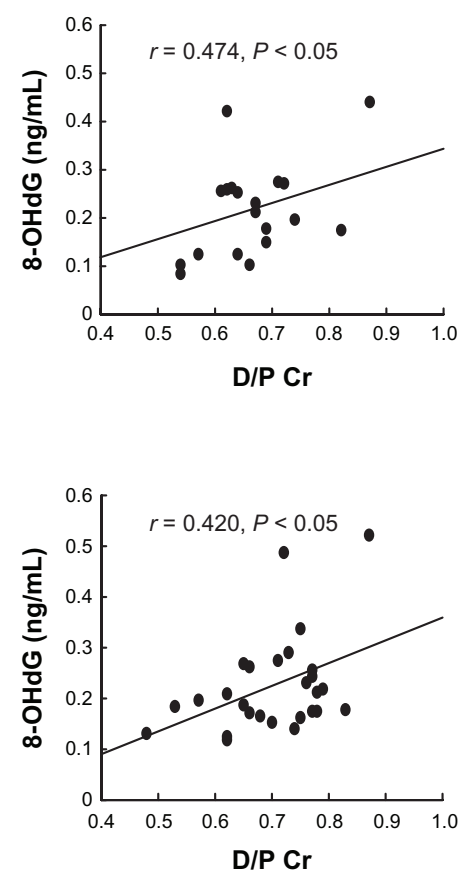
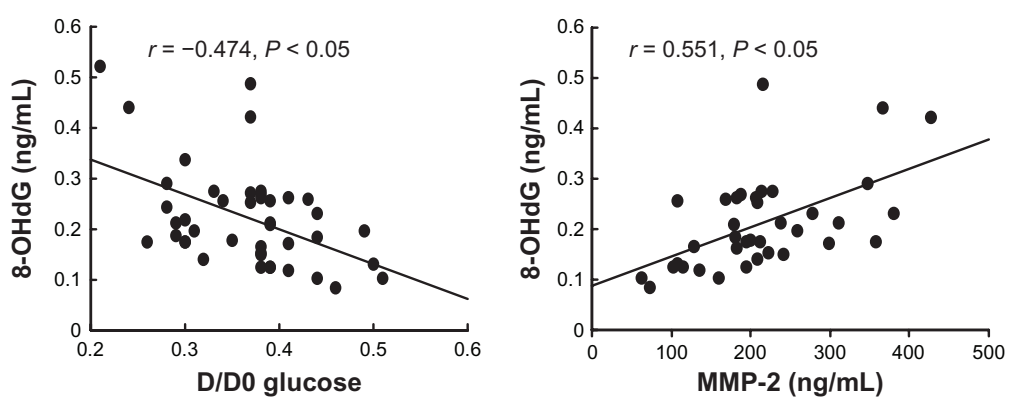

Peritonitis history (+)
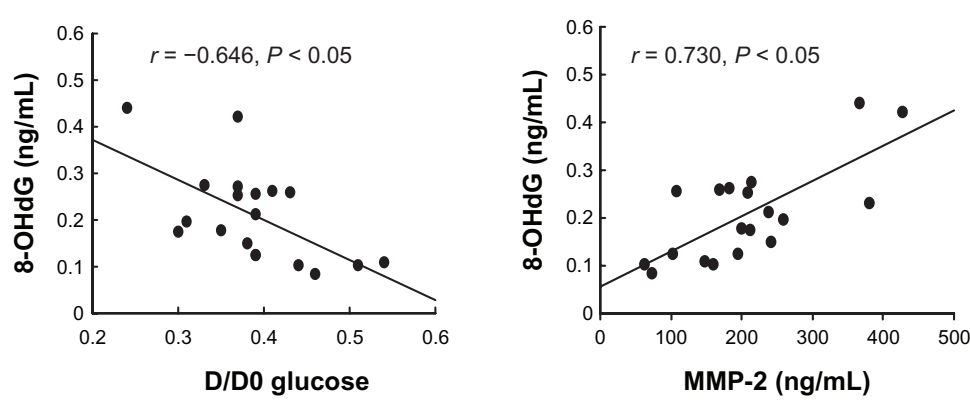

Peritonitis history (-)
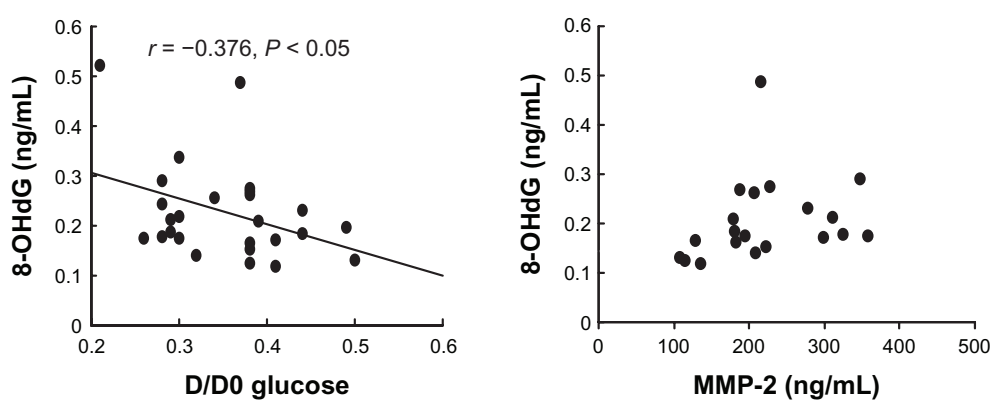

Figure 2 Correlation between the 8-hydroxydeoxyguanosine (8-OHdG) level in drained dialysate and the peritoneal solute transport rate, determined by peritoneal equilibration test and matrix metalloproteinase-2 (MMP-2) level in drained dialysate: 8-OHdG level and (A) dialysate:plasma creatine ratio (D/P Cr), D/D0 glucose (where D is the glucose level of peritoneal effluents obtained 4 hours after the injection and D0 is the glucose level obtained immediately after the injection), and MMP-2 level in peritoneal dialysis (PD) patients and (B) D/P Cr, D/D0 glucose, and MMP-2 level in those PD patients who had a past history of peritonitis and in those PD patients who did not.

MMP-2 level in the drained dialysate $(r=0.551, P<0.05)$ (Figure 2A). In the subanalysis based on past history of peritonitis, 8-OHdG level in the drained dialysate correlated with $\mathrm{D} / \mathrm{P} \mathrm{Cr}$ and inversely correlated with D/D0 glucose in both the $\mathrm{PD}$ patients who had a past history of peritonitis $(\mathrm{D} / \mathrm{P} \mathrm{Cr}$ : $r=0.474, P<0.05$; D/D0 glucose: $r=-0.646, P<0.05)$ and the $\mathrm{PD}$ patients who did not have a past history of peritonitis (D/P Cr: $r=0.420, P<0.05$; D/D0 glucose: $r=-0.376$, $P<0.05$ ) (Figure 2B). On the other hand, 8-OHdG level in the drained dialysate was significantly correlated with MMP-2 level in drained dialysate in the PD patients who had a past history of peritonitis $(r=0.730, P<0.05)$; however, it was not correlated with MMP-2 level in drained dialysate in those PD patients who did not have past history of peritonitis (Figure 2B).

\section{Characteristics of 8-OHdG concentration} in drained dialysate in PD patients

The 8-OHdG level in drained dialysate was not correlated with the age of subjects or the duration of PD in PD patients (Figure 3A). The 8-OHdG in drained dialysate was also not correlated with the SBP or DBP in PD patients (Figure 3B).

\section{Discussion}

The results of this study show that $8-\mathrm{OHdG}$ level in drained dialysate was significantly correlated with the solute transport rate, which was determined by PET. In addition, 8-OHdG level in drained dialysate was not correlated with age of PD patients, their duration of $\mathrm{PD}$, or their blood pressure (BP). These results suggested that $8-\mathrm{OHdG}$ level in drained 

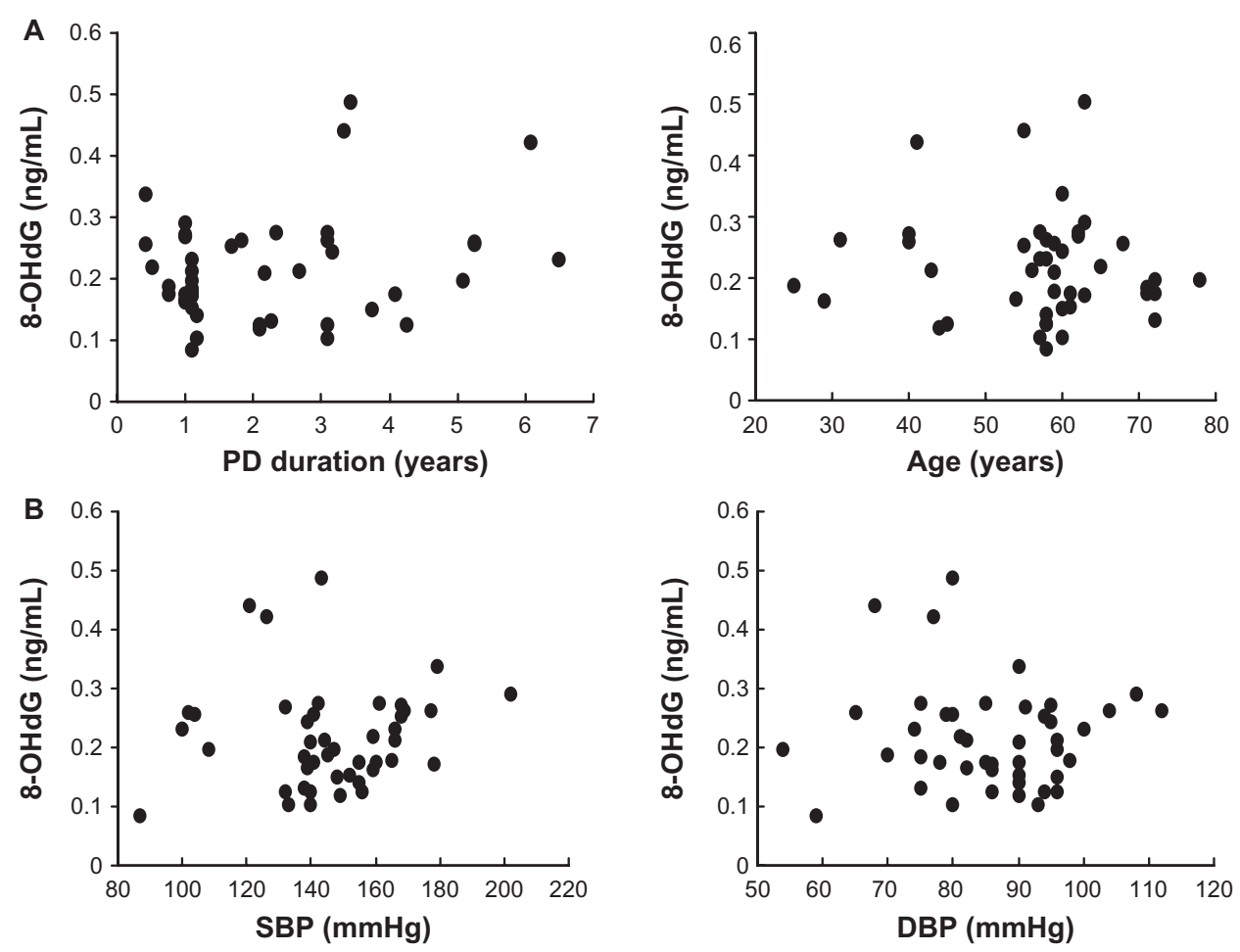

Figure 3 Correlation between the 8-hydroxydeoxyguanosine (8-OHdG) level in drained dialysate and the peritoneal dialysis (PD) duration and age of subject: 8-OHdG level and (A) PD duration and age of subject and (B) systolic blood pressure (SBP) and diastolic blood pressure (DBP).

dialysate reflects peritoneal damage and could be a novel marker of peritoneal damage. This is the first study to demonstrate a significant correlation between $8-\mathrm{OHdG}$ level in drained dialysate and peritoneal damage in PD patients. The oxidative stress marker $8-\mathrm{OHdG}$ is one of the most abundant oxidative products of DNA. Several studies have reported that $8-\mathrm{OHdG}$ is a useful marker for assessment of oxidative DNA damage by ROS, including that in end-stage renal disease patients. ${ }^{17,18}$

The mechanisms involved in peritoneal structural and functional changes remain unclear; however, several studies have reported that ROS contributed to multiple factors inducing peritoneal damage. ${ }^{13,14,19}$ Noh et $\mathrm{al}^{13}$ reported that increase of omental transforming growth factor beta-1 (TGF- $\beta_{1}$ ), vascular endothelial growth factor (VEGF), and type I collagen by conventional PD solution was blocked by an antioxidant, $\mathrm{N}$-acetylcysteine, in an animal model. Kihm et $\mathrm{al}^{14}$ also reported that increase of TGF- $\beta_{1}$, alpha smooth muscle actin, VEGF, and vessels per area in peritoneum by PD fluid was attenuated by an antioxidant, benfotiamine, in an animal model. They also reported interleukin (IL)-6- and CD3-positive cells per area in peritoneum were increased by PD fluid and that this increased IL-6 was blocked by benfotiamine. ${ }^{14}$ Gotloib et $\mathrm{al}^{19}$ reported that oxidative stress induced peritoneal fibrosis and sclerosis characterized by peritoneal adhesions, wrapping of intestinal loops, and the presence of a layer of fibrous tissue in animal PD model. These results suggest that ROS contribute to inflammation, fibrosis, revascularization, and sclerosis in PD.

Cancer antigen 125 (CA125) level can be considered as a marker for mesothelial cell mass and is often used as a marker of peritoneal injury. ${ }^{20-22}$ Although the downward trend with time of CA125 level in effluent suggested mesothelial cell mass, a single low value is difficult to interpret. MMP-2 level in drained dialysate has been reported to be associated with solute transport rate estimated with PET. ${ }^{16}$ Furthermore, MMP-2 level in effluent has been reported to be markedly increased in patients with peritoneal injury including EPS. ${ }^{15}$

In this study of PD patients, 8-OHdG levels in drained dialysate were similar between those who had a past history of peritonitis and those who did not. In addition, these levels were significantly correlated with $\mathrm{D} / \mathrm{P} \mathrm{Cr}$ and inversely correlated with D/D0 glucose, regardless of past history of peritonitis. On the other hand, 8-OHdG level in the drained dialysate was significantly correlated with MMP-2 level in drained dialysate in the PD patients who had a past history of peritonitis; however, it was not correlated with MMP-2 level in drained dialysate in those who did not have a past history of peritonitis. These results suggest that past history 
of peritonitis may contribute to peritoneal injury by association with oxidative stress, and that $8-\mathrm{OHdG}$ level in drained dialysate may be more strongly correlated with peritoneal injury in PD patients who have a past history of peritonitis than in PD patients who do not, beyond patients' PD duration, age, and BP. These results also suggest that inhibition of oxidative stress in the peritoneum may be a good therapeutic option to prolong PD duration, especially in PD patients with a past history of peritonitis.

A limitation of this study was that the plasma 8-OHdG level of each sample was not measured to investigate which of local production of 8-OHdG in the peritoneum or increased leakage of plasma 8-OHdG into the dialysate was associated with peritoneal injury in PD patients. Further large and long-term studies will be needed to elucidate the production and regulation of 8-OHdG and the association between 8-OHdG levels in drained dialysate and the development of peritoneal injury including EPS in PD. As it stands, 8-OHdG levels in drained dialysate could be used in combination with PET, MMP-2, and CA125 level in drained dialysate to evaluate peritoneal injury.

\section{Conclusion}

In conclusion, the results of the present study suggest that 8-OHdG level in drained dialysate may be a useful novel marker of peritoneal damage in PD.

\section{Disclosure}

The authors report no conflicts of interest in this work.

\section{References}

1. Krediet RT. Advances in peritoneal dialysis. Minerva Urol Nefrol. 2007; 59(3):251-260.

2. Burkart J, Piraino B, Kaldas H, et al. Why is the evidence favoring hemodialysis over peritoneal dialysis misleading? Semin Dial. 2007;20(3): 200-202.

3. Van Biesen W, Vanholder R, Lameire N. The role of peritoneal dialysis as the first-line renal replacement modality. Perit Dial Int. 2000;20(4):375-383.

4. Hung KY, Huang JW, Tsai TJ, Chen WY. Natural changes in peritoneal equilibration test results in continuous ambulatory peritoneal dialysis patients: a retrospective, seven year cohort survey. Artif Organs. 2000; 24(4):261-264.

5. Williams JD, Craig KJ, Topley N, et al. Morphologic changes in the peritoneal membrane of patients with renal disease. J Am Soc Nephrol. 2002;13(2):470-479.
6. Davies SJ, Phillips L, Naish PF, Russell GI. Peritoneal glucose exposure and changes in membrane solute transport with time on peritoneal dialysis. J Am Soc Nephrol. 2001;12(5):1046-1051.

7. Gandhi VC, Humayun HM, Ing TS, et al. Sclerotic thickening of the peritoneal membrane in maintenance peritoneal dialysis patients. Arch Intern Med. 1980;140(9):1201-1203.

8. Kawanishi H. Encapsulating peritoneal sclerosis. Nephrology (Carlton). 2005;10(3):249-255.

9. Kawaguchi Y, Saito A, Kawanishi H, et al. Recommendations on the management of encapsulating peritoneal sclerosis in Japan, 2005: diagnosis, predictive markers, treatment, and preventive measures. Perit Dial Int. 2005;25 Suppl 4:S83-S95.

10. Twardowski ZJ, Nolph KD, Khanna R. Limitations of the peritoneal equilibration test. Nephrol Dial Transplant. 1995;10(11): 2160-2161.

11. Mortier S, Faict D, Schalkwijk CG, Lameire NH, De Vriese AS. Long-term exposure to new peritoneal dialysis solutions: effects on the peritoneal membrane. Kidney Int. 2004;66(3):1257-1265.

12. Lee HB, Yu MR, Song JS, Ha H. Reactive oxygen species amplify protein kinase $\mathrm{C}$ signaling in high glucose-induced fibronectin expression by human peritoneal mesothelial cells. Kidney Int. 2004; 65(4):1170-1179.

13. Noh H, Kim JS, Han KH, et al. Oxidative stress during peritoneal dialysis: implications in functional and structural changes in the membrane. Kidney Int. 2006;69(11):2022-2028.

14. Kihm LP, Müller-Krebs S, Klein J, et al. Benfotiamine protects against peritoneal and kidney damage in peritoneal dialysis. J Am Soc Nephrol. 2011;22(5):914-926.

15. Hirahara I, Inoue M, Okuda K, Ando Y, Muto S, Kusano E. The potential of matrix metalloproteinase-2 as a marker of peritoneal injury, increased solute transport, or progression to encapsulating peritoneal sclerosis during peritoneal dialysis: a multicentre study in Japan. Nephrol Dial Transplant. 2007;22(2):560-567.

16. Hirahara I, Inoue M, Umino T, Saito O, Muto S, Kusano E. Matrix metalloproteinase levels in the drained dialysate reflect the peritoneal solute transport rate: a multicentre study in Japan. Nephrol Dial Transplant. 2011;26(5):1695-1701.

17. Tarng DC, Huang TP, Wei YH, et al. 8-hydroxy-2'-deoxyguanosine of leukocyte DNA as a marker of oxidative stress in chronic hemodialysis patients. Am J Kidney Dis. 2000;36(5):934-944.

18. Austin EW, Parrish JM, Kinder DH, Bull RJ. Lipid peroxidation and formation of 8-hydroxydeoxyguanosine from acute doses of halogenated acetic acids. Fundam Appl Toxicol. 1996;31(1):77-82.

19. Gotloib L, Wajsbrot V, Cuperman Y, Shostak A. Acute oxidative stress induces peritoneal hyperpermeability, mesothelial loss, and fibrosis. $J$ Lab Clin Med. 2004;143(1):31-40.

20. Rodrigues AS, Almeida M, Fonseca I, et al. Peritoneal fast transport in incident peritoneal dialysis patients is not consistently associated with systemic inflammation. Nephrol Dial Transplant. 2006; 21(3):763-769

21. Rodrigues AS, Martins M, Korevaar JC, et al. Evaluation of peritoneal transport and membrane status in peritoneal dialysis: focus on incident fast transporters. Am J Nephrol. 2007;27(1):84-91.

22. Van Esch S, Zweers MM, Jansen MA, de Waart DR, van Manen JG, Krediet RT. Determinants of peritoneal solute transport rates in newly started nondiabetic peritoneal dialysis patients. Perit Dial Int. 2004; 24(6):554-561

\section{Publish your work in this journal}

The International Journal of Nephrology and Renovascular Disease is an international, peer-reviewed open-access journal focusing on the pathophysiology of the kidney and vascular supply. Epidemiology, screening, diagnosis, and treatment interventions are covered as well as basic science, biochemical and immunological studies. The journal welcomes

\section{Dovepress}

original research, clinical studies, reviews \& evaluations, expert opinion and commentary, case reports and extended reports. The manuscript management system is completely online and includes a very quick and fair peerreview system, which is all easy to use. Visit http://www.dovepress.com/ testimonials.php to read real quotes from published authors. 